\title{
EVALUATION OF THE EFFECTIVENESS OF ANTERIOR COMBINED APPROACH OF 3 IN 1 BLOCK AND SCIATIC NERVE BLOCK BY SINGLE INJECTION TECHNIQUE FOR BELOW KNEE PROCEDURES
}

\author{
Arunachalam $R^{1}$, Venkatraghavan $C^{2}$, Sivakumar $G^{3}$ \\ ${ }_{1}^{1}$ Assistant Professor, Department of Anaesthesiology, Government Mohan Kumaramangalam Medical College Hospital, Salem, \\ Tamilnadu, India. \\ ${ }^{2}$ Assistant Professor, Department of Anaesthesiology, Government Mohan Kumaramangalam Medical College Hospital, Salem, \\ Tamilnadu, India. \\ ${ }^{3}$ Professor and HOD, Department of Anaesthesiology, Government Mohan Kumaramangalam Medical College Hospital, Salem, \\ Tamilnadu, India.
}

ABSTRACT
BACKGROUND
Regional Anaesthesia is the preferred anaesthetic technique for surgical procedures in the lower limb and among various
techniques, nerve blocks provide an interesting option.

\section{MATERIALS AND METHODS}

We studied the effectiveness of nerve locator guided anterior combined approach of 3 in 1 block and sciatic nerve block by single injection technique for below knee procedures.

\section{RESULTS}

We have evaluated the mean onset and duration of block in the distributions of the branches of the lumbar plexus and the sciatic nerve.

\section{CONCLUSION}

We conclude that the single injection technique is a viable and suitable option for anterior combined approach of 3 in 1 block and sciatic nerve blocks.

\section{KEYWORDS}

3 in 1 Block, Anterior Combined Approach.

HOW TO CITE THIS ARTICLE: Arunachalam R, Venkatraghavan C, Sivakumar G. Evaluation of the effectiveness of anterior combined approach of 3 in 1 block and sciatic nerve block by single injection technique for below knee procedures. J. Evolution Med. Dent. Sci. 2018;7(47):5089-5093, DOI: $10.14260 /$ jemds/2018/1131

\section{BACKGROUND}

Ever since the introduction of regional anaesthesia to the armamentarium of anaesthesia, it has been the preferred choice of anaesthesiologists, wherever applicable. Regional anaesthetic techniques score over general anaesthesia particularly in the management of patients with multiple comorbidities.

Among the regional anaesthesia techniques, Spinal anaesthesia is most chosen technique for lower limb procedures. However, since long, anaesthesiologists have shown keen interest in exploring the possibility of isolated nerve blocks for lower limb procedures ${ }^{1}$. Compared to the upper limb, plexus blocks and nerve blocks are more technically demanding in the lower limb. The deeper location of the nerve trunks and the fact that the nerve trunks of the lower limb nerve plexus are located wider apart are the reasons for this observation.

'Financial or Other Competing Interest': None.

Submission 03-10-2018, Peer Review 02-11-2018,

Acceptance 09-11-2018, Published 19-11-2018.

Corresponding Author:

Dr. Venkatraghavan C,

Assistant Professor

Department of Anaesthesiology,

Government Mohan Kumaramangalam

Medical College Hospital,

Salem, Tamilnadu, India.

E-mail: drvenkatraghavan76@gmail.com

DOI: $10.14260 /$ jemds $/ 2018 / 1131$

\section{(c) (i) $(9)$}

The use of nerve locators has largely contributed to the improved success of lower limb nerve blocks. In the present study we evaluated the effectiveness of anterior combined approach of 3 in 1 block by a single injection technique using nerve locators for below knee surgical procedures. This technique subverts the need for the usual multiple injection techniques employed for blocking the major nerves of the lower limb, namely femoral nerve, obturator nerve, sciatic nerve and several other cutaneous nerves. Moreover, in this technique patient can remain supine, adding comfort to the patient during the procedure, unlike other techniques involving other patient positions.

\section{Objective}

To evaluate the effectiveness of anterior combined approach of 3 in 1 block and sciatic nerve block by single injection technique, using nerve locator, for below knee surgical procedures.

\section{MATERIALS AND METHODS \\ Description of the Study}

The Prospective Observational Study proposal was submitted to the Institutional Ethics Committee of the Government Mohan Kumaramangalam Medical College Hospital, where the study was subsequently conducted. Approval of the ethics committee was given for the study. The study proposed to evaluate the effectiveness of anterior combined approach of 3 in 1 block and sciatic nerve block by single injection technique, using nerve locator, for below knee procedures. 
The study group was chosen from the patient population scheduled to undergo below knee surgical procedures like soft tissue surgeries of the leg and foot. 25 such patients belonging to ASA category I and II and age group more than 18 years were chosen for the study. During patient selection, those with bleeding disorders, any infection at local site, neurological and neuromuscular diseases, allergy to local anaesthetic diseases, severe renal and hepatic diseases, and pregnancy (Female patients) were carefully excluded.

The patients were subjected to basic investigations and pre-anaesthetic evaluation as per institution protocol. Prior informed consent was obtained from the patients participating in the study.

At the start of the procedure, intravenous access was established. Standard monitoring was done throughout the study period. All patients were sedated with Inj. Midazolam $0.03 \mathrm{mg} / \mathrm{Kg}$ prior to the study procedure.

At the start of the procedure, the following anatomical landmarks were identified and marked on the side of planned surgical procedure. A straight line was drawn from the anterior superior iliac spine to the pubic tubercle. This line was divided into 3 parts. Second line was drawn from the greater trochanter parallel to the above line. From the middle and medial third of the first line, a perpendicular was drawn to join the second line, marked as point B. The point of pulsation of the femoral artery lying over the first line was marked as point $\mathrm{A}$. Midpoint of the line $\mathrm{AB}$ was marked as point $\mathrm{C}$, which was chosen as the landmark for needle puncture.

Sterile precautions were instituted throughout the procedure. The needle puncture site was infiltrated with local anaesthetic, $2 \mathrm{ml}$ of Inj. Lignocaine hydrochloride 1\% using a $24 \mathrm{G}$ needle.

The type of nerve locator used for the procedure was supplied by Fischer and Paykel with model name "Innervators 232". $150 \mathrm{~mm}$ b-bevelled Stimuplex A (BBraun $^{\mathrm{TM}}$ ) was used for the procedure. Initially the nerve locator was set to deliver a pulse current of $0.6 \mathrm{~mA}$, frequency $1 \mathrm{~Hz}$ and duration $200 \mu \mathrm{sec}$.

The nerve blocks were performed as per the following procedure. Sciatic nerve block was performed first. The needle was directed $10^{\circ}$ laterally and $80^{\circ}$ caudally. At times, the needle might come close to the femoral nerve, indicated by appearance of patellar movement. If patellar movements stopped on its own, the needle was introduced further with the current of the nerve locator increased to $5 \mathrm{~mA}$. On the contrary, in case the patellar movement persisted even at 0.6 $\mathrm{mA}$, the insulated needle was reoriented medially by a small measure. At a depth of around $9 \mathrm{~cm}$, the sciatic nerve was identified by the stimulation of either of its components. The common peritoneal component stimulation was indicated by appearance of dorsiflexion or eversion of foot, whereas, the stimulation of the tibial component was indicated by plantar flexion or inversion of the foot and flexion of the toes. Thereafter, the needle orientation was optimally adjusted to ensure the same stimulation response at a current intensity of 0.4 to $0.6 \mathrm{~mA}$. After ensuring negative aspiration for blood, $15 \mathrm{ml}$ of $0.25 \%$ bupivacaine was injected at $5 \mathrm{ml}$ aliquots.

With the needle at the same point of insertion, it was withdrawn upto the level of subcutaneous tissue and redirected $45^{\circ}$ in the cephalad direction and $10^{\circ}$ medially towards point $A$, until patellar movements were observed.
The current intensity in the nerve locator was decreased to $0.4 \mathrm{~mA}$ and the needle adjusted to get a stimulation response. After ensuring negative aspiration for blood, $30 \mathrm{ml}$ of $0.25 \%$ bupivacaine was injected, with pressure applied manually distally to facilitate cephalad spread of the drug.

Following the injections, the quality of block was evaluated using Hollmen's scale. Sensory block was assessed across 4 grades $(0-$ Normal pin prick; + Pin prick felt, but weak compared to the other extremity; ++ Pin prick felt as mild touch; +++ No perception). Motor blockade was also assessed across 4 grades (Grade 0- Normal Muscle function; + Minimal depression in muscle power compared to the state before the block; ++ Very weak residual muscular function persisting in muscles; +++ Complete motor block)

The nerves studied included Sensory nerves (Femoral, Obturator, Lateral femoral cutaneous, Tibial, Superficial peroneal, Deep peroneal, \& Posterior femoral cutaneous nerves) and motor nerves (Femoral, Obturator, Tibial, Common peroneal nerves).

The Onset time of the block and the duration of analgesia and motor block were noted across the distribution of the nerves under study. Quality of surgical anaesthesia was also observed. A 3-grade system was used (Excellent- No complaints of pain during surgery; Good- Minimal pain relieved by a small dose of intravenous Fentanyl; Poor- In case of severe pain necessitating the institution of general anaesthesia)

\section{Statistical Methods}

Calculation of the Mean and Standard Deviation of the onset and duration of sensory and motor block in the distribution of the Sciatic Nerve and Femoral Nerve

\section{RESULTS}

The age, weight and height distribution of patients involved in the study have been presented in figures.(1-3) The sciatic nerve at located at a depth of $9.6 \pm 3 \mathrm{~cm}$ (Figure 1) and the femoral nerve was found at a depth of $5.1 \pm 4 \mathrm{~cm}$ (Figure 2)

The mean onset of sensory block in the distribution of sciatic nerve was as follows: Tibial nerve- $31.76 \pm 2.78$ minutes; Superficial peroneal nerve 29.24 \pm 2.75 minutes; Deep peroneal nerve $29.45 \pm 6.51$ minutes; Posterior femoral cutaneous nerve $29.31 \pm 13.12$ minutes (Figure 3 ).

The mean onset of sensory block in the distribution of 3 in 1 block was as follows: Femoral nerve- 20.64 \pm 2.51 minutes; Lateral femoral cutaneous nerve- $23.45 \pm 8.91$ minutes; Obturator nerve- 23.19 \pm 6.76 minutes (Figure 4).

The mean onset of motor block in the distribution of sciatic nerve was as follows: Tibial nerve- 39.54 \pm 8.32 minutes; Common peroneal nerve $39.85 \pm 15.11$ minutes (Figure 5). The mean onset of sensory block in the distribution of 3 in 1 block was as follows: Femoral nerve$29.70 \pm 7.21$ minutes; Obturator nerve- $31.95 \pm 12.81$ minutes (Figure 6).

The mean duration of sensory block was $352.6 \pm 41.01$ minutes in the distribution of sciatic nerve block and was $413.4 \pm 48.8$ minutes in the distribution of 3 in 1 block (Figure 7). The mean duration of motor block was $316.4 \pm 43.80$ minutes in the distribution of sciatic nerve block and was $332.91 \pm 40.91$ minutes in the distribution of 3 in 1 block (Figure 8). 
Overall, the quality of the nerve blocks during the surgical procedure was "Excellent" in 92\% of cases; Good in 2\% and Poor in $2 \%$ of cases. In cases where the operating conditions were poor with patients complaining of pain (1 out of the 25 cases), General anaesthesia was supplemented with.

One case of accidental vascular puncture was observed during needle steering towards the sciatic nerve. It was conservatively managed.

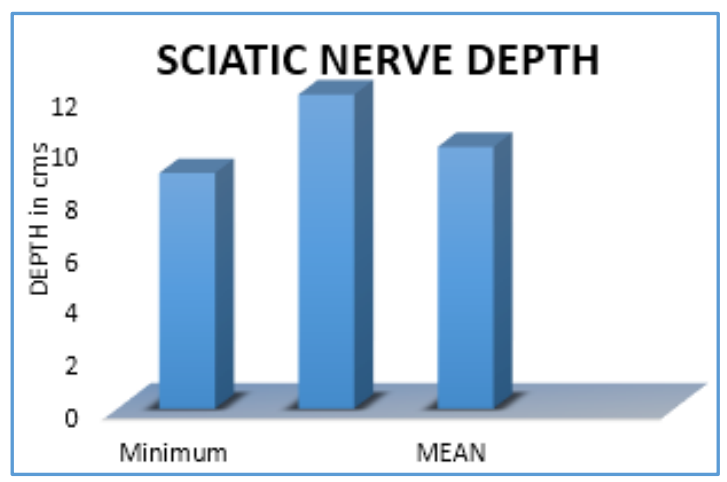

Figure 1

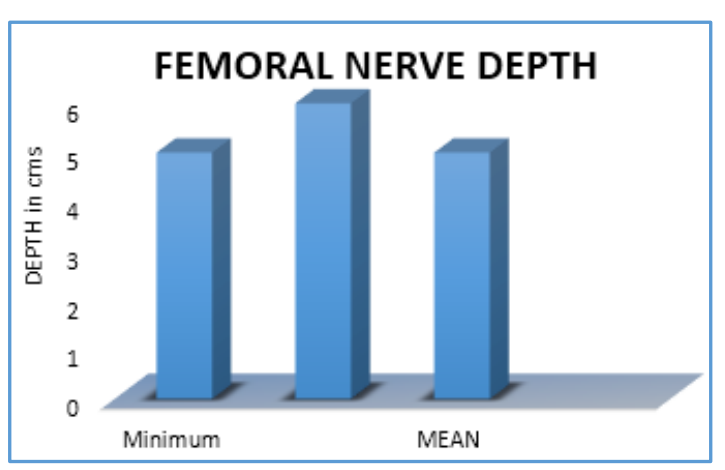

Figure 2

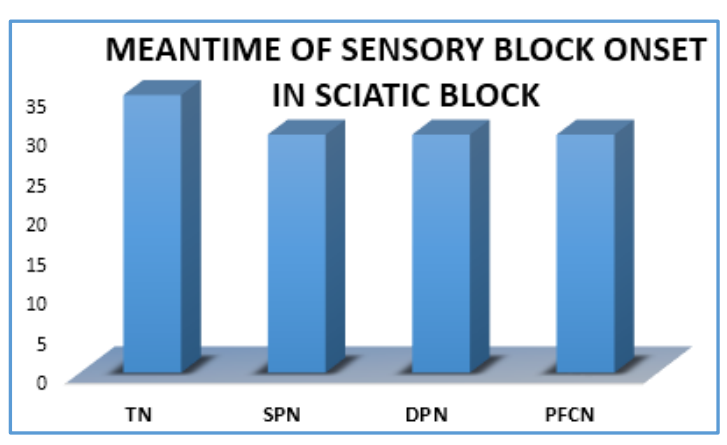

Figure 3

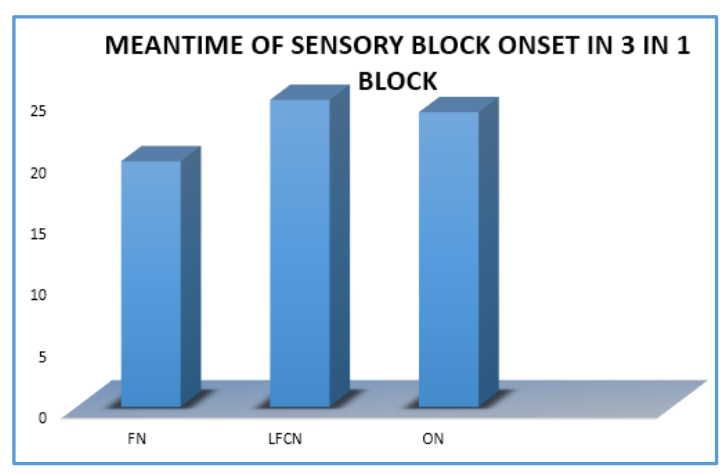

Figure 4

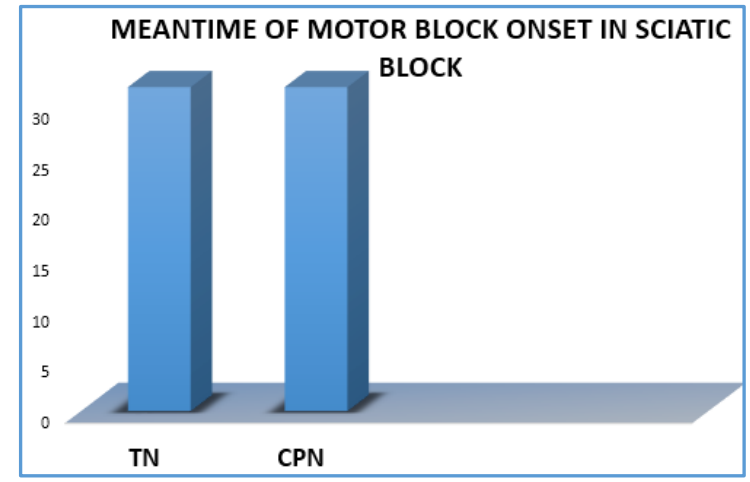

Figure 5

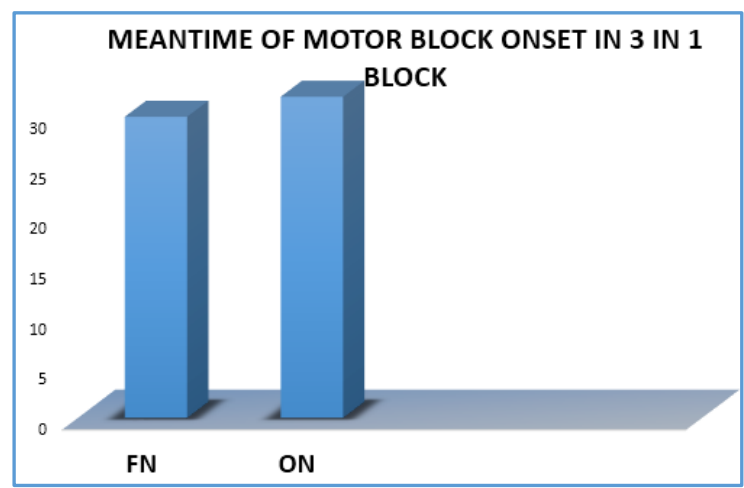

Figure 6

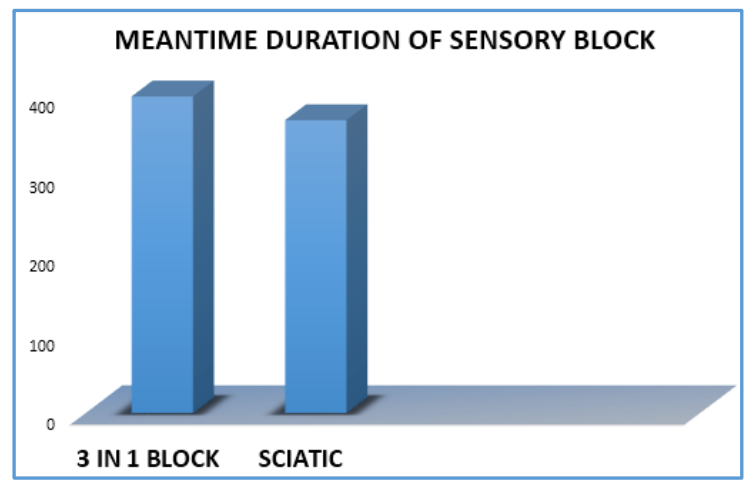

Figure 7

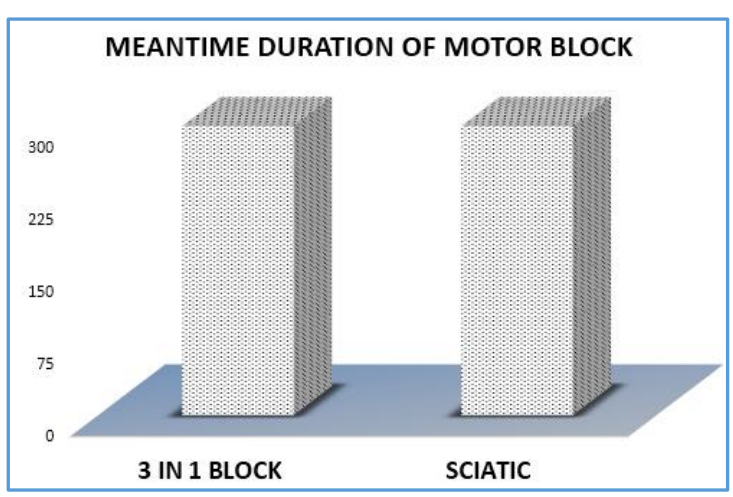

Figure 8

\section{DISCUSSION}

Regional anaesthetic techniques have been the preferred option for anaesthetizing patients presenting for lower limb procedures. Apart from Subarachnoid block, combining lumbar plexus and sciatic nerve blocks has been a technique of prolonged interest to anaesthesiologists. 
In 1887, Crile ${ }^{1}$ performed lower limb amputations by exposing the sciatic nerve in the gluteal fold and femoral nerve in the inguinal fold and administering cocaine in the intraneural sheath.

While nerve plexus block in the upper limb is being extensively performed, similar technique for the lower limb remains elusive due to certain anatomical considerations. George Mirshel ${ }^{2}$ had noted that lack of a sheath like covering for the lumbar plexus makes it difficult to perform a successful nerve plexus block like the axillary brachial plexus block in the upper limb.

Another observation by Dogliotti $\mathrm{AM}$ et $\mathrm{al}^{3}$ noted that the nerve trunks of the lumbar plexus are wide apart and hence there is a need for multiple injections to achieve a successful blockade. The deeper location of the nerve plexus also meant difficulty in reaching the nerve plexus thereby resulting often in incomplete anaesthesia and frequent failure of blocks.

Despite the proposed drawbacks, there has been a sustained interest towards performing peripheral nerve blocks for operations on the lower leg and foot. Bridenbaugh et $\mathrm{al}^{4}$ in a study authoritatively stated that peripheral nerve block is the anaesthesia of choice for lower limb surgeries.

In a landmark study, Winnie AP, Ramamurthy S, Durrani ${ }^{5}$ described the anatomy of the lumbar plexus. They found that the lumbar plexus is sandwiched between the psoas major muscle and the ilacus muscles. They described the fascial compartment surrounding the lumbar plexus above and below the inguinal ligament. They performed lumbar plexus block by the inguinal perivascular approach using these anatomical considerations. Applying a distal pressure to the point of injection causes a cephalad spread and blocks the femoral, obturator and lateral femoral cutaneous nerves resulting in the classically described " 3 in 1 " block. In the present study this technique was employed in performing the "3 in 1" block.

For performing sciatic nerve block, various techniques have been described. Gaston Labat ${ }^{6}$ described the classical posterior approach wherein, the patient was turned into the sim's position to perform the block. Though this technique has a very high success rate (99\%), patient positioning to perform the block is difficult in many cases.

Lateral approach to perform sciatic nerve block was performed, albeit with lesser success rate (80\%), by Ichiyanagi. 7

George $\mathrm{P} \mathrm{Beck}^{8}$ described the anterior approach to sciatic nerve block. The main advantage claimed was that the block can be performed with the patient in supine position. Success percentage was also high (95\%). The needle insertion point corresponds to point B in figure A new landmark for anterior approach has been mentioned in a study by Chelley et al. ${ }^{9}$ However, in more than half of the cases, the posterior cutaneous nerve was spared.

Carole Barbero et al ${ }^{10}$ proposed a formula based on the height of the patient to determine the landmark for needle insertion. In the same study, the authors preferred to perform sciatic nerve block first, and then perform the 3 in 1 block to avoid injury to the femoral nerve. This approach of performing sciatic nerve block first was followed in our study. A new single position supine approach to sciatic and femoral nerve block was described by Raj et al.11 However the lithotomy position which they described was difficult to be achieved in patients with limited mobility.
Pierre Pandin et al ${ }^{12}$ proposed the single injection technique for the combined 3 in 1 block and sciatic nerve block. From the needle injection point described, the needle was directed caudad and laterally to perform the sciatic nerve block; and then directed cephalad and medially to locate the femoral nerve. This approach was successfully employed in our study.

In the present study, in the " 3 in 1 " block, femoral nerve was blocked in $100 \%$ of cases; Obturator nerve in $92 \%$ cases; lateral femoral cutaneous nerve was blocked in $84 \%$ of cases. Hence a true " 3 in 1" block was occurred only in $84 \%$ of the patients. However, with a high success of femoral and saphenous nerve block achieved in this technique, surgeries on the leg and foot were possible without any further supplementation of anaesthetics.

Blockade of sciatic nerve block resulted in $100 \%$ block of Tibial and Superficial Peroneal nerves; deep peroneal nerve block in $96 \%$ of patients; and posterior cutaneous nerve of thigh block in only $76 \%$ of patient. For leg and foot surgeries, the blockade of the first three nerves mentioned above is found sufficient.

\section{CONCLUSION}

We found that the above findings correlate well with previous studies done by Pierre Padin et al. The single injection combined " 3 in 1" block with sciatic nerve block is found to be sufficient for leg and foot surgeries. The nerve branches spared in the above blocks are those which supply the regions of the lower limb above the knee.

The time of onset of sensory block of sciatic nerve block was almost 20 to 30 minutes. This delayed onset is most likely due to the large size of the nerve, necessitating more diffusion of the local anaesthetic solution. Studies done by Coventry et $\mathrm{al}^{13}$ and Chelly et al have described similar findings of delayed onset of sciatic nerve block.

\section{REFERENCES}

[1] Bridenbaugh PO. The lower extremity: somatic blockade. In: Cousins MJ, Bridenbaugh PO, eds. Neural blockade in clinical anesthesia and management of pain. $2^{\text {nd }}$ edn. Philadelphia, PA: JB Lippincott 1988: p. 417-36.

[2] Hirschel G. Textbook of Local anaesthesia. New York: William Wood \& Company 1914.

[3] Dogliotti AM. Anaesthesia: narcosis- local regional spinal. Chicago: SB Debour 1939.

[4] Bridenbaugh LD. Lower extremity nerve blocks. Int Anaesthesia Clinics 1963;1(3):745-56.

[5] Winnie AP, Ramamurthy S, Durrani Z. The inguinal para-vascular technic of lumbar plexus anaesthesia: the "3-in-1" block. Anesth Analg 1973;52(6):989-96.

[6] Labat G. Regional Anaesthesia- its technical and clinical applications. $2^{\text {nd }}$ edn. Philadelphia: WB Saunders Company 1923.

[7] Ichiyanagi K. Sciatic nerve block: lateral approach with the patient supine. Anesthesiology 1959;20:601-4.

[8] Beck GP. Anterior approach to sciatic nerve block. Anesthesiology 1963;24:222-4.

[9] Chelly JE, Delaunay L. A new anterior approach to the sciatic nerve block. Anesthesiology 1999;91(6):165560 . 
[10] Barbero C. Anterior approach to sciatic nerve blockadaptation to the patient's height: regional anaesthesia. Anesthesia and Analgesia 2004;98:19858.

[11] Raj PP, Parks RI, Watson TD, et al. A new singleposition supine approach to sciatic-femoral nerve block. Anaesthesia and Analgesia 1975;54(4):489-93.
[12] Pandin P, Vancutsem N, Salengros JC, et al. The anterior combined approach via a single skin injection site allows lower limb anaesthesia in supine patients. Canadian Journal of Anaesthesiology 2003;50(8):8014.

[13] Coventry DM, Todd JG. Alkalinisation of bupivacaine for sciatic nerve blockade. Anaesthesia 1989;44(6):467-70. 